\title{
Folliculitis Decalvans Mimicking Frontal Fibrosing Alopecia
}

Natassia Soares Pizani ${ }^{\star}$, Marcelo de Souza Teixeira, Maria Fernanda Reis Gavazzoni Dias, Wellington Batista Vasques and Luciana Pantaleao

Department of Dermatology, Universidade Federal Fluminense, Brazil

*Corresponding author: Natassia Soares Pizani, Department of Dermatology, Universidade Federal Fluminense, Brazil, Tel: 5521983494195; E-mail: dra.natassia.pizani@gmail.com

Received date: August 04, 2017; Accepted date: January 18, 2018; Published date: January 23, 2018

Copyright: (c) 2018 Pizani NS, et al. This is an open-access article distributed under the terms of the Creative Commons Attribution License, which permits unrestricted use, distribution, and reproduction in any medium, provided the original author and source are credited.

\begin{abstract}
Folliculitis decalvans (FD) is a neutrophilic scarring alopecia with peripheral spreading alopecic patches with pustules, crusting and tufted hair. Frontal fibrosing alopecia (FFA) is a lymphocyte-mediated primary cicatricial alopecia that affects frontal hairline and eyebrows. We report a 27 -year-old black male presenting scarring alopecia with symmetrical retreat of the frontal and temporal borders of the hair implantation and eyebrow thinning. Skin biopsy revealed compact orthokeratosis, corneal plugs, regular acanthosis, superficial and deep perivascular and periadnexal inflammatory infiltrate, that was compatible with FD. We present a case of FD with retreat of hair line implantation and thinning of the eyebrows, as it is seen in FFA.
\end{abstract}

Keywords: Alopecia; Scalp dermatoses; Hair follicle; Dermoscopy

\section{Introduction}

Folliculitis Decalvans (FD) is a chronic pustular eruption of the scalp that results in permanent patchy alopecia [1]. It predominantly occurs in young and middle-aged adults with a slight preference for male gender. Biopsy specimens of early lesions reveal follicular neutrophilic abscesses in the infundibula or upper levels of the follicle. Late lesions show dermal lymphocytes, follicles' destruction and dermal fibrosis.

Frontal fibrosing alopecia (FFA) is a variant of Lichen Planopilaris. Typically postmenopausal women present with a scarring process that starts on the frontal hairline and then gradually extends backwards. There may be associated eyebrow loss [1-7]. The advancing edge of the alopecic area may demonstrate minute perifollicular papules at the bases of terminal hairs. Typical pathology findings include interface dermatitis with necrotic keratinocytes, vacuolization of the basal lawyer and dense band-like lymphocytic infiltrate in the follicular infundibulum and isthmus [7].

\section{Case Report}

We present a 27-year-old black male showing scarring alopecia with symmetrical retreat of the frontal and temporal borders of the hair implantation and eyebrow thinning (Figure 1).

The clinical manifestations began 14 years ago and remain in progress. The borders of the hair line show erythema and perifollicular scaling with centripetal activity. The parietal area and vertex exhibit numerous tufted hairs with perifollicular scaling and erythema. Vertex and occipital area present alopecic patches with centrifugal activity. The patches have atrophic center, erythema, scaling, crusts and tufted hair on the border (Figure 2).

The dermoscopic findings are similar to FFA, since there is perifollicular scaling and erythema and no pustules at the current stage (Figure 3).
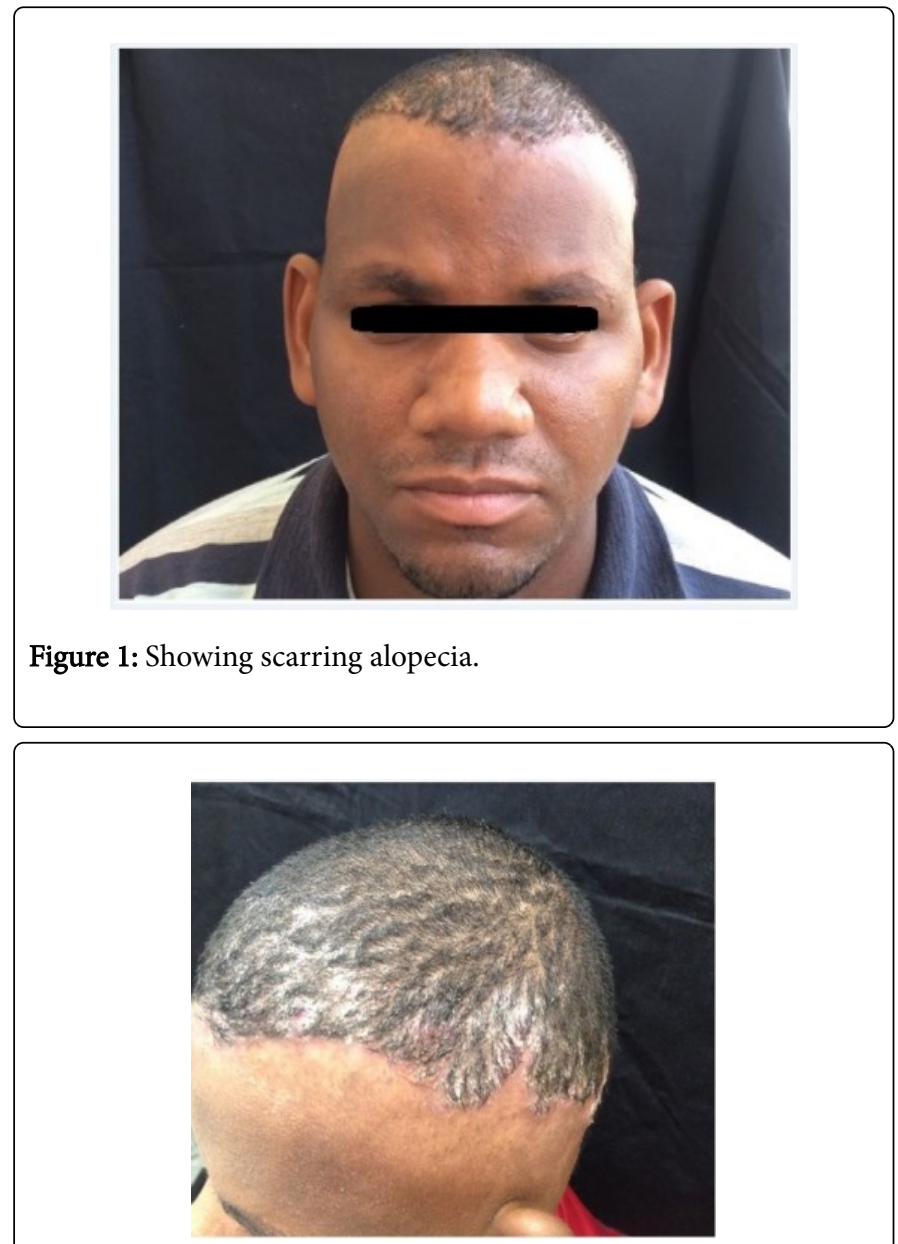

Figure 2: Showing the patches have atrophic center, erythema, scaling, crusts and tufted hair on the border. 
Page 2 of 2

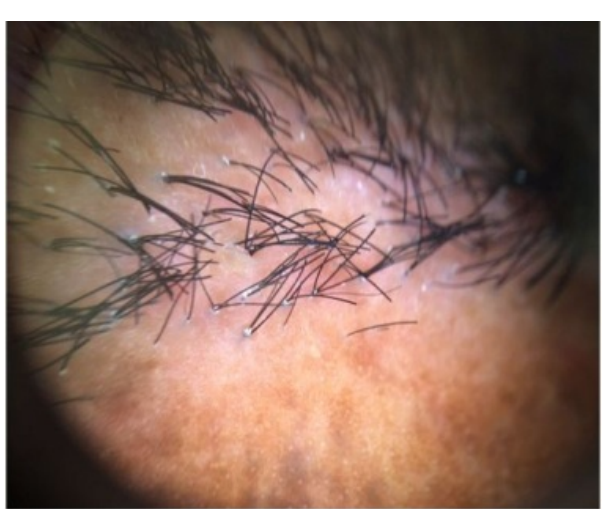

Figure 3: Showing perifollicular scaling and erythema and no pustules.

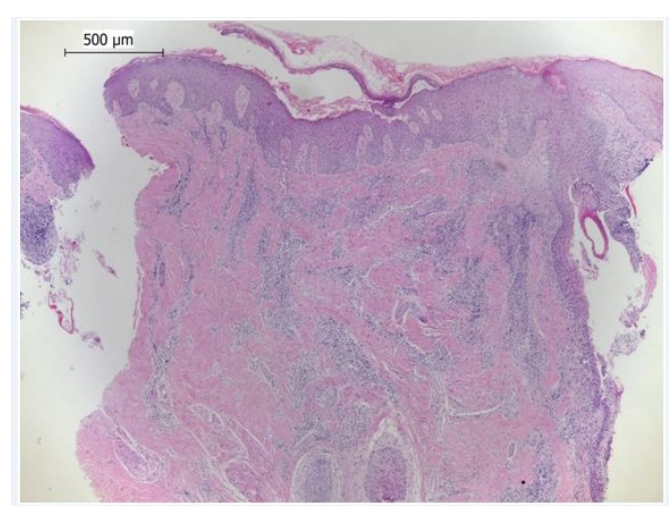

Figure 4: Superficial and deep perivascular and periadnexal inflammatory infiltrate.

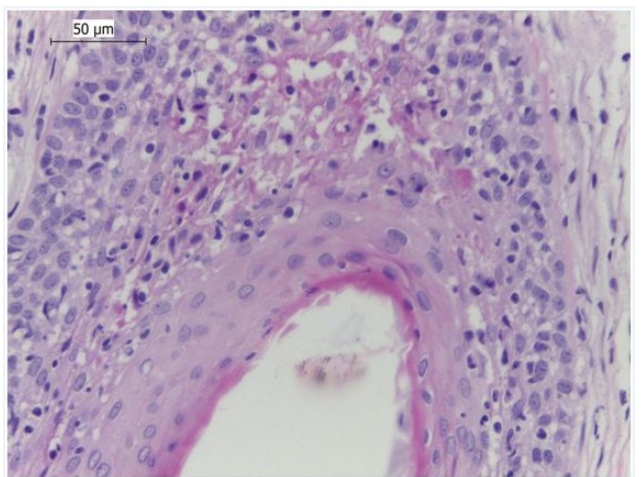

Figure 5: Folliculitis is present besides numerous plasmocytes, Russell bodies and extensive fibrosis.

The histopathology shows compact orthokeratosis with corneal plugs, regular acanthosis, superficial and deep perivascular and periadnexal inflammatory infiltrate (Figure 4). Folliculitis is present besides numerous plasmocytes (Figure 5) Russell bodies and extensive fibrosis. This is compatible with FD.

\section{Discussion}

The cicatricial alopecias include a broad group of disorders characterized by permanent destruction of the hair follicle and irreversible hair loss. Among other features, cicatricial alopecias can be classified according to the inflammatory infiltrate [2]. FFA is characterized by the development of frontotemporal loss of both terminal and vellus hairs in a band-like distribution. Eyebrow involvement occurs in 50-83 percent of patients [2]. Histologically, there is an infiltrate predominantly lymphocytic. FD is characterized by one or more confluent areas of scarring alopecia and multiple pustules, with or without inflammatory papules at the periphery of alopecia patches. It may involve any area of the scalp and multifocal involvement on the scalp is common [3]. The appearance of quiescent or partially treated FD may present only with scarring alopecia and perifollicular erythema or scale. At this stage, clinical distinction between FD and primary lymphocytic cicatricial alopecias, like FFA, can be difficult.

Fernandez-Crehuet et al. performed a retrospective study about dermoscopic findings of FD in 58 patients. In conclusion, tufted hairs, perifollicular erythema, and follicular hyperkeratosis are the hallmarks trichoscopic features of FD [1]. According to Rossi et al. the main trichoscopic features of FFA are absence of follicular openings, scarring white patches, minor perifollicular scaling, perifollicular erythema, and lone hairs [8].

In the reported case, the patient presented a longstanding condition, about 13 years of evolution. Initially the clinical feature was a typical FD. Recently the appearance became closer to FFA due to the progression in the frontotemporal area.

Dermoscopic findings such as perifollicular erythema and scaling, absence of follicular openings and tufted hair combined features from FD and FFA. Despite the clinical presentation similar to FFA, the histopathological analysis was compatible with FD.

The goal of this article is to demonstrate an unusual presentation of folliculitis decalvans and therefore expand diagnostic hypothesis considering scarring alopecia with similar clinical presentation but different therapeutic approach.

\section{References}

1. Fernández-Crehuet $\mathrm{P}$, Vañó-Galván S, Molina-Ruiz AM, RodriguesBarata AR, Serrano-Falcón C, et al. (2017) Trichoscopic Features of Folliculitis Decalvans: Results in 58 Patients. Int J Trichology 9: 140-141.

2. Mirmirani P, Willey A, Headington JT, Stenn K, McCalmont TH, et al. (2005) Primary cicatricial alopecia: histopathologic findings do not distinguish clinical variants. J Am Acad Dermatol 52: 637-643.

3. AlGaadi S, Miteva M, Tosti A (2015) Frontal Fibrosing Alopecia in a Male Presenting with Sideburn Loss. Int J Trichology 7: 72-73.

4. Vañó-Galván S, Molina-Ruiz AM, Serrano-Falcón C, Arias-Santiago S, Rodrigues-Barata AR, et al. (2014) Frontal fibrosing alopecia: a multicenter review of 355 patients. J Am Acad Dermatol 70: 670-678.

5. Tan E, Martinka M, Ball N, Shapiro J (2004) Primary cicatricial alopecias: clinicopathology of 112 cases. J Am Acad Dermatol 50: 25-32.

6. MacDonald A, Clark C, Holmes S (2012) Frontal fibrosing alopecia: a review of 60 cases. J Am Acad Dermatol 67: 955-961.

7. Contin LA, de Almeida Ledá YL, Caldeira Nassif K, Suárez Restrepo MV (2017) Patchy Frontal Fibrosing Alopecia: Description of an Incomplete Clinical Presentation. Skin Appendage Disord 3: 190-192.

8. Rossi A, Grassi S, Fortuna MC, Garelli V, Pranteda G, et al. (2017) Unusual patterns of presentation of frontal fibrosing alopecia: A clinical and trichoscopic analysis of 98 patients. J Am Acad Dermatol 77: 172-174. 